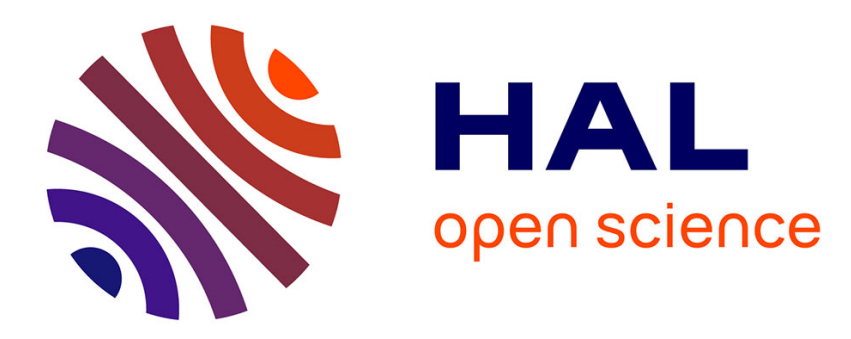

\title{
Détermination expérimentale des éléments principaux d'un système optique
}

A. Cornu

\section{To cite this version:}

A. Cornu. Détermination expérimentale des éléments principaux d'un système optique. J. Phys. Theor. Appl., 1877, 6 (1), pp.276-282. 10.1051/jphystap:018770060027601 . jpa-00237305

\section{HAL Id: jpa-00237305 https://hal.science/jpa-00237305}

Submitted on 1 Jan 1877

HAL is a multi-disciplinary open access archive for the deposit and dissemination of scientific research documents, whether they are published or not. The documents may come from teaching and research institutions in France or abroad, or from public or private research centers.
L'archive ouverte pluridisciplinaire HAL, est destinée au dépôt et à la diffusion de documents scientifiques de niveau recherche, publiés ou non, émanant des établissements d'enseignement et de recherche français ou étrangers, des laboratoires publics ou privés. 


\title{
DÉTERMINATION EXPÉRIMENTALE DES ÉLÉMENTS PRINGIPAUX D'UN SYSTEME OPTIQUE;
}

\author{
Par M. A. CORNU.
}

La détermination des éléments principaux d'un système optique ne présente théoriquement aucune difficulté : au point de vue pratique, il n'en est pas de même lorsqu'on veut opérer avec précision; on se trouve la plupart du temps en présence de cas particuliers, dans lesquels les règles ordinaires ne s'appliquent plus ou du moins présentent dans leur application des circonstances inadmissibles.

Ayant rencontré un certain nombre de ces difficultés, à l'occasion de recherches diverses, je me suis attaché à les résoudre le plus simplement qu'il m'a été possible de le faire.

\section{1. - Détermination des éléments principaux d'un objectif composé quelconque.}

Il s'agit, comme on sait, de fixer, sur l'axe principal du système, la position de quatre points : les deux foyers principaux et les deux points nodaux (images du centre optique).

La règle que je vais indiquer s'applique aussi bien à une lentille simple qu'à un système de lentilles, qui est, comme on le sait, équivalent à une lentille unique. Il est bon d'employer une lumière lomogène, à moins que le système ne soit bien achromatique; l'emploi d'un verre coloré suffit souvent pour donner une grande précision aux pointés; mais il est nécessaire, tout d'abord, de vérifier que le système est aplanétique, c'est-à-dire que tous les rayons qu'il doit réfracter concourent bien à la formation d'une image unique dans tous les cas où l'on devra l'utiliser; s'il n'en était pas ainsi, en réduisant son ouverture par un diaphragme de grandeur et de position convenables, on arriverait à le rendre suf- 
fisamment aplanétique pour posséder les propriétés théoriques qui servent de définition aux éléments principaux.

Ces propriétés sont les suivantes : les foyers principaux $\mathrm{F}, \mathrm{F}^{\prime}$ $(f i g . I)$ sont les points de convergence des rayons parallèles à l'axe du système; les points nodaux $\mathbf{N}, \mathbf{N}^{\prime}$ sont les images virtuelles du centre optique, vues par un observateur placé d'un côté ou de l'autre du système : les distances $\mathrm{NF}$ et $\mathrm{N}^{\prime} \mathbf{F}^{\prime}$ sont égales à la distance focale principale $f$, et les distances $x, x^{\prime}$ de deux foyers con-

Fig. $\mathrm{I}$.

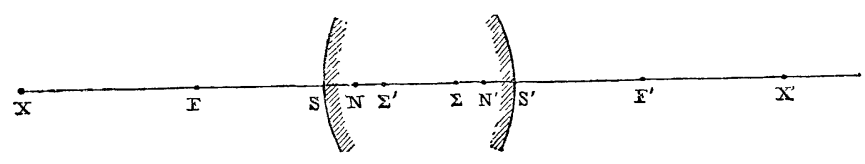

jugués quelconques $\mathrm{X}, \mathrm{X}^{\prime}$ aux foyers principaux correspondants

( $\left.{ }^{1}\right)$ Il faut apporter quelque attention dans certains systèmes optiques pour bien établir la correspondance des trois points $\mathrm{X}, \mathrm{F}, \mathrm{N}$ et $\mathrm{X}^{\prime}, \mathrm{F}^{\prime}, \mathrm{N}^{\prime}$, car, suivant les cas, les points nodaux $\mathrm{NN}^{\prime}$ et les points $\mathrm{FF}^{\prime}$ peurent occuper les positions relatives les plus diverses.

On évitera toute erreur en commençant par supposer une source de lumière réelle $\mathrm{X}$, située à l'infini, du côté gauche par exemple, et se déplaçant toujours dans le même sens (vers la droite), jusqu'à la première surface (celle de gauche du système): on aurà $x=-\infty$. On supposera l'observateur placé de l'autre côté du sysstème, et marquant successivement les foyers conjugués qu'il rencontrera : d'abord le foyer principal $\mathrm{F}^{\prime}$, e'est l'orıgine des $x^{\prime}$; le point lumineux se rapprochant, le foyer conjugué $x^{\prime}$ s'eloiggnera de $\mathrm{F}^{\prime}$ et $x^{\prime}$ croîtra positivement; quand le point lumineux sera arrêté au point $\mathrm{F}$, tel que $\mathrm{X}^{\prime}$ soit à l'infini, la distance $x$ sera nulle, car $\mathrm{F}$ est l'origine de $x$. Le point lumineux $X$ continuant sa marche, le foyer conjugué $\mathrm{X}^{\prime}$ ne sera plus réel : l'observateur ne juge de la position de ce foyer que par la divergence des rayons émergुents : on dit qu'alors le foyer conjugué est virtuel, il a passé à gauche du système; il faut donc compter $x$ négativement par rapport aussi à $F^{\prime}$ qui est son origine, tandis que $x$ est positif, compté relativement à $\mathrm{F}$. Le point lumineux s'avançant toujours arrivera à la première surface (celle de gauche); là, matériellement, il ne pourrait plus avancer; mais, pour l'observateur placé de l'autre côté du sỳstème, tout se passera comme si la source lumineuse pénétrait réellement dans le système, si l'on prend pour source lumineuse le point de concours de rayons concentrés par un système optique quelconque : de cette manière le point lumineux peut atteindre jusqu'au centre optique, c'est-à-dire jusqu'à un point tel que tout rayon entrant par la première surface du système émerge parallèlement à sa direction à sa sortie de la dernière. L'image virtuelle du centre optique pour l'observateur, toujours situé du côté de la dernière surface, est le point nodal $\mathrm{N}^{\prime}$. Les trois points $\mathrm{N}^{\prime}, \mathrm{F}^{\prime}, \mathrm{X}^{\prime}$ correspondants sont donc ainsi bien définis. On obtiendrait de même la correspondance des trois autres. 
sont liées entre elles par la relation

$$
x x^{\prime}=-f^{2},
$$

On met le signe - si l'on compte $x$ et $x^{\prime}$ dans le même sens.

La règle ordinaire s'en déduit aisément : $\mathbf{1}^{\circ}$ on détermine la position des foyers principaux $F$ et $\mathrm{F}^{\prime}$, en tournant successivement les faces du système vers un objet situé à l'infini, et l'on observe de l'autre côté du système le foyer des rayons conjugués; $2^{\circ}$ on place un objet à une distance $x$ d'un des foyers principaux et on observe la distance $x^{\prime}$ du foyer conjugué à l'autre foyer principal; le produit $x x^{\prime}$ donne le carré $f^{2}$ de la distance focale principale cherchée.

I $^{\circ}$ Détermination des foyers principaux. - Là déjà on rencontre une petite difficulté pratique; on dispose rarement d'un objet situé assez loin pour qu'on puisse le considérer comme à l'infini; on peut le remplacer par un collimateur, mais il faut que ce collimateur ait lui-même été réglé : la difficulté n'est donc que déplacée. L'emploi d'un miroir plan, utilisé sous l'incidence normale, simplifie sous certains rapports ce réglage; mais on peut s'en passer à l'aide de la règle suivante, qui n'est pas neuve, mais qui n'est pas assez connue :

On vise un objet assez éloigné, situé à $m$ fois la distance focale de l'objectif, $x=m f$; la distance $x$ ' du foyer conjugué au foyer principal est la fraction $\frac{\mathbf{1}}{m}$ de la longueur focale principalef.

C'est une conséquence de la formule $x x^{\prime}=-f^{2}$ qu'on peut écrire $\frac{x^{\prime}}{f}=-\frac{f}{x} ;$ si donc $x=m f$, il vient $x^{\prime}=-\frac{f}{m} ;$ comme on le voit, la distance de l'objet est comptée non pas à partir du centre de l'objectif, mais à partir du foyer principal F situé du côté opposé à l'observateur; mais, comme on doit prendre $m$ assez grand pour pouvoir confondre $\frac{\mathrm{I}}{m}$ avec $\frac{\mathbf{I}}{m+1}$, il suffit de connaître d'une manière approchée la distance de l'objet.

Ainsi, avec un objectif de $\mathrm{o}^{\mathrm{m}}$, 3o de distance focale, un objet situé à $30 o^{m}$ donne un foyer conjugué, qui ne diffère que de la fraction $\frac{0,3}{300}$ ou $\frac{1}{1000}$ de la distance focale $0^{\mathrm{m}}, 300$, c'est-à-dire de $\frac{1}{3}$ de millimètre. 
On peut donc se contenter d'observer avec exactitude la position $\mathrm{du}$ foyer de l'objet à $3 \mathrm{oo}^{\mathrm{m}}$ : la correction à faire subir à cette observation est déterminée avec une exactitude parfaitement suffisante, quand bien même la distance de l'objet ne serait qu'imparfaitement déterminée. Une graduation sur le tirage des appareils optiques employés permet d'effectuer cette correction avec une extrême facilité. Le sens de la correction n'est jamais douteux : si, par suite d'une complication quelconque, on hésitait sur ce sens, il suffirait d'observer successivement des objets de plus en plus éloignés : la position des foyers successifs de ces objets indiquera le sens de la correction qui détermine le foyer de l'infini.

Ainsi, avec cette règle, on peut se passer d'objets situés à de grandes distances, de collimateur ou de miroir plan, et inversement on peut régler les collimateurs et contrôler la planéité d'un miroir.

$2^{\circ}$ Détermination des points nodaux. - Il faut que $x$ et $x^{\prime}$ soient déterminés avec une précision suffisante, car $f^{2}=-x x^{\prime}$; dès lors, si l'on commet une erreur $\delta x$ sur $x$ et $\delta x^{\prime}$ sur $x^{\prime}$, on commet sur $f$ une erreur $\delta f$, telle que

$$
-(f+\delta f)^{2}=(x+\delta x)\left(x^{\prime}+\delta x^{\prime}\right)
$$

ou approximativement

$$
-2 f \delta f=x \delta x^{\prime}+x^{\prime} \delta x
$$

divisant par $f^{2}$ ef remarquant que

il vient

$$
\frac{x}{f^{2}}=-x^{\prime} \quad \text { et } \quad \frac{x}{f^{2}}=-x
$$

$$
\frac{\delta f}{f}=\frac{1}{2}\left(\frac{\delta x^{\prime}}{x^{\prime}}+\frac{\delta x}{x}\right),
$$

d'où l'on conclut que l'erreur relative commise sur la distance focale est la moyenne des erreurs relatives commises sur les coordonnées $x$ et $x^{\prime}$ des foyers conjugués.

On reconnaît que ni $x$ ni $x^{\prime}$ ne doivent être très-petits, car ces quantités entrent en dénominateur, et, comme $\delta x$ et $\delta x^{\prime}$ ne peuvent pas être nulles, l'erreur relative augmente rapidement avec la petitesse de $x$. 
On pourrait croire que $\delta x$ peut être égal à zéro ou au moins négligeable, puisque la position de l'objet peut être définie avec une grande exactitude; mais il n'en est rien, car $x$ est la différence entre la position de l'objet et la position du foyer $\mathrm{F}$; comme cette dernière est nécessairement erronée de $\delta \mathrm{F}, x$ est au moins égal à $\delta \mathrm{F}$.

En tout cas, il est évident que, si $x$ et $x^{\prime}$ sont de mème ordrc de grandeur, $\delta x^{\prime}$ et $\delta x$ sont aussi de mème ordre; pour trouver les meilleures conditions à remplir, on peut supposer $\delta x^{\prime}=\delta x==\delta \mathrm{F}$, c'est-à-dire égale à l'erreur qu'on commet dans la détermination du foyer principal; on aura donc

$$
\frac{f}{\partial f}=\frac{\partial \mathrm{F}}{2}\left(\frac{\mathrm{I}}{x}+\frac{\mathrm{I}}{x^{\prime}}\right) \quad \text { avec } \quad x x^{\prime}=f^{2} \text {. }
$$

Les valeurs de $x$ et $x^{\prime}$ qui rendent $\delta f$ minimum sont évidemment $x=x^{\prime}$, car on peut écrire

$$
\frac{\delta f}{f}=-\delta \mathbf{F} \frac{x+x^{\prime}}{2 x x^{\prime}}=\frac{\delta \mathbf{F}}{2} \frac{x+x^{\prime}}{f^{2}}
$$

il faut donc que les distances focales conjuguées soient voisines de l'égalité.

On est tenté alors de choisir pour la position de $\mathrm{X}$ et $\mathrm{X}^{\prime}$ les points situés au double de la distance focale principale, comme dans le focomètre de Silbermann; mais cette disposition a l'inconvénient pratique d'exiger souvent, pour l'appareil de mesure, un développement inadmissible, égal au quadruple de la distance focale.

J'ai pensé qu'il était beaucoup plus pratique et surtout plus exact de prendre pour $x$ et $x^{\prime}$ deux valeurs, pour ainsi dire égales et de signe contraire à celles-là, à savoir deux points très-voisins des points nodaux: les deux systèmes de points que je recommande, en raison des simplifications qu'ils apportent, sont les sommets des surfaces extérieures du système optique donné et leurs images observées à travers la surface opposée.

A cet effet, on trace (avec un pinceau et de l'encre de Chine délayée dans un peu d'eau gommée) sur le milieu de l'une des surfaces un petit trait $\mathrm{S}$, et l'on mesure, par un moyen qui sera indiqué plus loin, la distance $\varepsilon^{\prime}$ de son image $\Sigma^{\prime}$, vue à travers la șurface $S^{\prime}$, 
au sommet $\mathrm{S}^{\prime}$, sur laquelle on a tracé également un petit trait $(f i g . \mathrm{I})$.

Comme on a mesuré préalablement les distances des foyers principaux $\mathrm{F}$ et $\mathrm{F}^{\prime}$ aux surfaces voisines $\mathrm{SS}^{\prime}$, à savoir $\mathrm{FS}=d, \mathrm{~F}^{\prime} \mathrm{S}^{\prime}=d^{\prime}$, on voit que, si $\mathrm{S}$ sert de point lumineux, $\Sigma^{\prime}$ est son foyer conjugué; dès lors on a

$$
d\left(d^{\prime}+\varepsilon^{\prime}\right)=-f^{2} .
$$

Mais, comme on peut de la même manière, en retournant le système, observer $\mathrm{S}^{\prime}$ à travers la surface $\mathrm{S}$, c'est-à-dire son image $\Sigma$ et mesurer la distance $\mathrm{S} \Sigma=\varepsilon$, on aura une nouvelle équation

$$
d^{\prime}(d+\varepsilon)=-f^{2},
$$

laquelle fournit une vérification d'autant plus précieuse qu'elle est très-aisée à obtenir. On détermine ainsi de deux manières la distance focale principale $f$.

Connaissant la valeur de $f$, en la portant en sens convenable, à partir de $\mathbf{F}$ et de $\mathrm{F}^{\prime}$ sur l'axe principal, on détermine alors les points nodaux $\mathrm{NN}^{\prime}$.

L'équation de vérification peut se mettre sous la forme intéressante

$$
d \varepsilon^{\prime}=d^{\prime} \varepsilon \quad \text { ou } \quad \frac{d}{d^{\prime}}=\frac{\varepsilon^{\prime}}{\varepsilon},
$$

qui s'interprète aisément.

La détermination de $\varepsilon$ et $\varepsilon^{\prime}$ se fait très-aisément : il suffit de placer le système proposé sur un petit chariot mobile le long d'une règle divisée; un microscope à long foyer, muni d'un réticule el d'un oculaire positif convenable, permet de définir avec précision le plan de visée en $\varphi$. On déplace le système optique de façon à amener successivement dans le plan de visée ol le trait de la surface $\mathrm{S}^{\prime}$ et l'image du trait $\mathrm{S}$, c'est-à-dire sur le réticule l'image du trait de la surface $\mathrm{S}^{\prime}$, puis l'image conjuguée du trait $\mathrm{S}$; la différence des lectures du chariot dans ces deux positions donne la longueur $\varepsilon^{\prime}$.

On peut même, si la course du chariot est suffisante, déterminer aussi $d^{\prime}$ par une troisième lecture : il suffit de disposer dans l'axe même de l'appareil un objet suffisamment éloigné (ou un collimateur au besoin), et de déplacer le chariot jusqu'à ce que le plan principal du système optique vienne coüncider avec le plan focal $\varphi$ du microscope, ce que l'observateur déterminera par la conJ. de Phys., t. VI. (Septembre 1877.) 
dition de voir l'image redressée de l'objet en coïncidence avec le plan du réticule.

Les trois lectures consécutives du chariot donnent donc $d$ et $d^{\prime}+\varepsilon$; si l'on recommence les mêmes opérations en retournant le système optique, on obtiendra de même $d$ et $d+\varepsilon$, par suite, les éléments de la détermination de $f^{2}$.

Je pense que la solution du problème proposé est arrivée à son maximum de simplicité pratique.

$$
\text { (A suivre.) }
$$

